Society. We suggest, however, that this first report of the use of a marker gas during manual aspiration of spontaneous pneumothorax has provided information about the likely short term outcome that cannot reliably be determined on the basis of the postaspiration chest radiograph alone and that this information may guide the clinician as to whether or not tube drainage is likely to be required.

We thank Dr D G Bell for helpful advice, Ms Wendy Armstrong and $\mathrm{Mr}$ John Lee for technical help, and Mrs Brenda Cooke for typing the manuscript.
1 Seaton A, Seaton D, Leitch AG. Pneumothorax. In: Crofton and Douglas's respiratory diseases. Oxford: Blackwell, 1989:761-83.

2 Raja OG, Lalor AJ. Simple aspiration of spontaneous pneumothorax. Brf Dis Chest 1981;75:207-8.

3 Hamilton AAD, Archer GJ. Treatment of pneumothorax by simple aspiration. Thorax 1983;38:934-6.

4 Jones JS. A place for aspiration in the treatment of spontaneous pneumothorax. Thorax 1985;40:66-7.

Bevelaqua FA, Aranda C. Management of spontaneous pneumothorax with small lumen cathether manual aspiration. Chest 1982:81:693-4.

6 Coady TJ, Seaton D, Day A, Bell DG, Carter A. Measurement of exhaled chlorofluorocarbons. Lancet 1988;ii:1286-7.

7 Cummings JH. The colon: physiology. In: Pounder RE, ed. Recent advances in gatroenterologv 7. Edinburgh: Churchill Livingstone, 1988:328.

(Accepted 12 November 1990)

\title{
Usefulness of immunotherapy in patients with severe summer hay fever uncontrolled by antiallergic drugs
}

\author{
V A Varney, M Gaga, A J Frew, V R Aber, A B Kay, S R Durham
}

Department of Allergy and Clinical Immunology, National Heart and Lung Institute, Royal Brompton and National Heart and Lung Hospital, London SW3 6LY

V A Varney, MRCP, research fellow

M Gaga, MD, research fellow

A J Frew, MRCP, lecturer

A B Kay, FRCP, professor

S R Durham, MRCP, senior

lecturer

Department of Clinical Epidemiology, National Heart and Lung Institute, Royal Brompton and National Heart and Lung Hospital, London

SW3 6LY

V R Aber, MSc, lecturer

Correspondence to: Dr S R Durham, Allergy Clinic, Royal Brompton and National Heart and Lung

Hospital, London SW3 6HP

BMf 1991;302:265-9

\section{Abstract}

Objective-To evaluate the efficacy and safety of immunotherapy (hyposensitisation) in patients with severe summer hay fever.

Design-A randomised, double blind, placebo controlled study of a biologically standardised depot grass pollen extract.

Setting-Allergy clinic, Royal Brompton and National Heart Hospital, London.

Patients -40 adults (mean age 35 years) with a history of severe grass pollen allergy uncontrolled by standard antiallergic drugs. Patients with perennial asthma were specifically excluded.

Intervention-Patients were randomised to receive either an active preparation (Alutard SQ, a grass pollen (Phleum pratense) extract) or placebo at a rate of two subcutaneous injections a week in increasing doses until a maintenance dose was reached. This maintenance dose was given once a month.

Main outcome measures-Clinical efficacy was evaluated by symptom and drug diary cards, visual analogue scores during the grass pollen season, and a postseasonal assessment by the patients and a doctor. Conjunctival and skin sensitivity to local allergen provocation was measured before and after eight months of treatment.

Results - There was a highly significant decrease (median Alutard SQ $v$ median placebo (95\% confidence interval for difference between medians)) in total symptom scores $(\mathbf{p}=\mathbf{0 . 0 0 1})$ in the Alutard SQ treated group (360 $v 928$ (238 to 825)). Significant differences were also found in total drug use $(\mathrm{p}=0.002,129 v 627(178$ to 574$))$. Visual analogue symptom scores were also reduced in the active group $(p=0.02,2.2 v 5.5(-4.8$ to -0.5$))$. The postseasonal assessment, by either the doctor or the patients, showed a large improvement $(p<0.001)$ in favour of Alutard SQ. Provocation tests showed a greater than 10-fold reduction for the active group in immediate conjunctival allergen sensitivity $(p=$ 0.001 ), a $40 \%$ decrease in early phase response $(p=0.02)$, and a $57 \%$ decrease in the late phase $(p=0.001)$ cutaneous response after intradermal allergen. A total of 523 active injections were given. There was one systemic reaction at 10 minutes after injection, which was rapidly reversed with intramuscular adrenaline. There was one mild delayed urticarial reaction at $2 \frac{1}{2}$ hours.

Conclusion-Immunotherapy is effective in patients with severe summer hay fever, but immediate anaphylactic reactions limit its use to specialised centres. Patient selection is extremely important, and chronic perennial asthma should be specifically excluded. As serious reactions occur within minutes a two hour wait for all patients after each injection seems unnecessary.

\section{Introduction}

Over the past 30 years there has been a substantial increase in the prevalence of summer hay fever in the United Kingdom. ${ }^{1}$ At the same time there have also been considerable improvements in treatment, particularly with the introduction of non-sedating selective histamine $\mathrm{H}_{1}$ antagonists ${ }^{2}$ and local nasal corticosteroids. Nevertheless, a minority of people with hay fever have extreme hypersensitivity to grass pollen and respond poorly to standard antiallergic drugs. In these people immunotherapy (hyposensitisation) would be the treatment of choice in many countries throughout the world, including the United States, Scandinavia, and the continent of Europe. ${ }^{34}$

In the United Kingdom allergen injection immunotherapy for treating disease mediated by IgE, including summer hay fever, has been largely discontinued on the recommendations of the Committee on Safety of Medicines in October 1986.5 The committee's report questioned the efficacy of immunotherapy and expressed concern about the number of deaths from severe bronchospasm and anaphylaxis. The committee recommended that injections should be given only where facilities for full cardiorespiratory resuscitation were immediately available, and that patients be kept under medical observation for at least two hours.

Generally, the committee's ruling was welcomed because it highlighted the potential dangers of immunotherapy, particularly in asthmatic patients. Nevertheless, the two hour waiting period has made this treatment impracticable for both patients and doctors.

For immunotherapy to retain a place in the treatment of summer hay fever we thought that a double blind, placebo controlled study in highly sensitive patients who were inadequately controlled by standard treatment was necessary in the United Kingdom. Previous studies have been described,,$^{6-8}$ but we are unaware of any double blind, placebo controlled studies using a biologically standardised extract. Such a study would re-evaluate the important issues of side effects and their timing along with efficacy. We chose the Alutard SQ vaccine as this is a biologically standardised depot preparation with a reported low incidence of systemic side effects and high efficacy. ${ }^{9-11}$ 


\section{Patients and methods}

PATIENTS

Forty patients were selected according to the following criteria: $(a)$ a history of severe summer hay fever, $(b)$ poor symptom control in previous years despite regular antiallergic treatment, which included antihistamines and intranasal corticosteroids and sodium cromoglycate, (c) a positive skin prick test result (weal diameter greater than $5 \mathrm{~mm}$ ) to timothy grass pollen extract (Phleum pratense, Soluprick, Allergologisk Laboratorium, Denmark). Patients were excluded if they gave an appreciable clinical history of other allergies, a history of immunotherapy in the previous five years, or a history of cardiovascular or other medical or immunological diseases. Patients with chronic asthma were excluded. Patients with mild seasonal asthma were included provided that their symptoms were controlled by inhaled $\beta$ sympathomimetics. All patients had normal or only slightly increased response of the airway to methacholinethat is, methacholine $\mathrm{PC}_{20}$ greater than $2 \mathrm{mg} / \mathrm{ml}\left(\mathrm{PC}_{20}\right.$ is the concentration of inhaled methacholine that caused a $20 \%$ decrease in one second forced expiratory volume). ${ }^{12}$ Clinical data on the patients are shown in table I.

TABLE I-Clinical data on patients

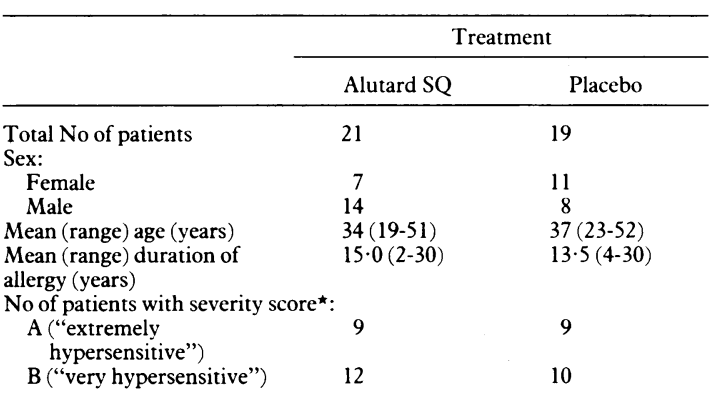

* Severity score $\mathrm{A}=$ skin prick test $>16 \mathrm{~mm}$; positive conjunctival provocation test at $<300$ biological units/ml; history of symptoms for $>10$ weeks associated with asthma and previous oral or injected corticosteroids, or both. Severity score $B=$ patients with moderate to severe hay fever who did not fulfil all of above criteria.

\section{STUDY DESIGN}

The study was performed with the approval of the Royal Brompton and National Heart Hospital ethics committee and had the patients' written informed consent. The patients were divided into two grades $\mathrm{A}$ and $B(A=$ extremely hypersensitive and $B=$ very hypersensitive) according to the severity of their symptoms (expressed as their severity score) and their skin and conjunctival response to allergen (table I). Patients in each grade were then randomised separately to active and placebo groups to ensure an even distribution of disease severity. Random allocation was performed by using computer generated random numbers. The groups were well matched for age and duration of disease. The coordinator, who was "blinded," was in charge of patient supervision and the adjustment of rescue treatment according to symptoms. She also performed objective (skin and conjunctival) and subjective tests before and after the pollen season. The immunotherapy injections were given by two doctors (operators) who had experience in this treatment and knew whether the patients were receiving either active or placebo preparations. Thus any reactions were seen and reported by the doctor who gave the injections and not by the blinded coordinator.

\section{IMMUNOTHERAPY PROTOCOL}

The active (Alutard SQ) and placebo treatment packs were identical. The placebo was $0.9 \%$ sodium chloride. During the induction phase this was randomly "spiked" with $0.01 \mathrm{mg} / \mathrm{ml}$ of histamine acid phosphate (in phosphate buffered saline, $\mathrm{pH} \mathrm{7 \cdot 4).} \mathrm{This} \mathrm{concen-}$ tration had previously been shown to produce local erythema and itching. A single batch of Alutard SQ (Allergologisk Laboratorium, Denmark) a partially purified and standardised extract of Phleum pratense was used throughout the study..$^{13}$ This extract is aluminium adsorbed for slow release. ${ }^{9 \cdot 11}$ Subcutaneous injections were started in April 1989 at a rate of two injections a week. The schedule of doses is shown in table II. Adjustments in the schedule were made on an

TABLE II - Immunotherapy schedule of twice weekly injections

\begin{tabular}{lccc}
\hline $\begin{array}{c}\text { Standardised quality } \\
\text { Injection No } \\
\text { units of Alutard/ml* }\end{array}$ & $\begin{array}{c}\text { Volume } \\
(\mathrm{ml})\end{array}$ & $\begin{array}{c}\text { Dose } \\
\text { (biological units) }\end{array}$ \\
\hline 1 & 100 & $0 \cdot 1$ & 3 \\
2 & 100 & $0 \cdot 5$ & 15 \\
3 & 1000 & $0 \cdot 1$ & 30 \\
4 & 1000 & $0 \cdot 4$ & 120 \\
5 & 1000 & $0 \cdot 8$ & 280 \\
6 & 10000 & $0 \cdot 1$ & 300 \\
7 & 10000 & $0 \cdot 3$ & 900 \\
8 & 10000 & $0 \cdot 5$ & 1500 \\
9 & 10000 & $0 \cdot 8$ & 2400 \\
10 & 100000 & $0 \cdot 1$ & 3000 \\
11 & 100000 & $0 \cdot 2$ & 6000 \\
12 & 100000 & $0 \cdot 4$ & 12000 \\
13 & 100000 & $0 \cdot 6$ & 18000 \\
14 & 100000 & $0 \cdot 8$ & 24000 \\
15 & 100000 & $1 \cdot 0$ & 30000 \\
\hline
\end{tabular}

$\star 100000 \mathrm{SQ} / \mathrm{ml}=30000$ biological units $/ \mathrm{ml}$ Phleum pratense.

individual basis. If patients developed a delayed local reaction of greater than $8 \times 8 \mathrm{~cm}$ the same dose was repeated at the next attendance. Injections were postponed if the patients were unwell. After 28 May we did not try any further increases in dose. All patients (active and placebo) had their maintenance dosesthat is, the volume of solution injected-reduced during the pollen season by $40 \%$. The maintenance dose was given once a month. The observation period was two hours after each injection.

\section{ASSESSMENTS}

All patients recorded daily symptom scores and drug requirements from April to October. The severity of individual symptoms was assessed on a scale of $0-3$ for chest (breathlessness, coughing, wheezing, and tightness), nose (sneezing, blocking, and running), eyes (itching, redness, streaming, and swelling), and mouth and throat symptoms (itching and dryness). Antiallergic drugs in the form of sodium cromoglycate eye drops and nasal spray were allowed freely, along with the short acting antihistamine acrivastine $(8 \mathrm{mg}$ every 6-8 hours as required) and the inhaled $b$ agonist, salbutamol. Nasal corticosteroids were not allowed. Patients were asked to use drugs regularly to control symptoms. If symptoms developed that were uncontrolled by regular drugs the coordinator could prescribe a seven day course of oral prednisolone $(30 \mathrm{mg}, 30 \mathrm{mg}$, $25 \mathrm{mg}, 20 \mathrm{mg}, 15 \mathrm{mg}, 10 \mathrm{mg}$, and $5 \mathrm{mg}$ ). Diary cards were scored by totalling the symptoms individually for each week, giving a maximum possible score of 21 for each symptom. Drugs were scored as follows: each eye drop, nasal spray, or inhalation of salbutamol scored 1 , and each acrivastine $(8 \mathrm{mg})$ or prednisolone $(5 \mathrm{mg})$ tablet scored 2.

During the season each patient was asked at two weekly intervals to indicate his or her overall hay fever symptoms as a visual analogue score (scale of 0-10) in response to the question, "How has your hay fever been this week?"

At the end of the season the coordinator and patients independently made an overall assessment of their hay fever compared with previous years using the following scale: +3 , much better; +2 , better; +1 , a little better; 0 , no change; -1 , a little worse; -2 , worse; -3 , a lot worse. 
Skin tests were performed on the extensor aspect of the forearm with an aqueous extract of Phleum pratense (Aquagen SQ; ALK, Denmark). Tests were performed before (March 1989) and after the season (November 1989) and at the same time of day for each patient. The size of the immediate (15 minute) and late phase (six and 24 hours) cutaneous responses to 30 biological units of intradermal allergen $(0.02 \mathrm{ml})$ and diluent $(0.02 \mathrm{ml})$ were assessed in duplicate. Reaction sizes were recorded in two dimensions, from which the mean diameter was calculated.

Conjunctival tests were performed before and after the pollen season according to the method of Moller $e t$ al. ${ }^{14}$ Half $\log$ incremental concentrations from 1 biological unit $/ \mathrm{ml}$ to 100000 biological unit $/ \mathrm{ml}$ were used; the Aquagen diluent $(0.03 \%$ human serum albumin, $0.5 \%$ phenol in $0.9 \%$ sodium chloride) was used as control.

\section{POLLEN COUNTS}

Daily grass pollen counts were obtained from a Burkard spore trap on the roof of the Polytechnic of North London (courtesy of Miss Jane Norris-Hill, pollen research project). Except for three patients, the group spent their working day in and around central London, where the counts obtained were relevant to their individual exposures.

\section{ANALYSIS OF RESULTS}

All data were collected double blind with respect to the clinical protocol and analysed by using a statistical package (Minitab). The results for placebo and actively treated groups were compared using a non-parametric test (Mann-Whitney U test ${ }^{15}$ ) or Fisher's exact test ${ }^{16}$ for one analysis only.

\section{Results}

A total of 37 (93\%) patients completed the study. The three patients who dropped out were from the placebo group. In two this was due to family problems and in the third delayed systemic reactions (shortness of breath) several hours after placebo injections. These withdrawals were therefore unlikely to have biased the statistical analysis. All 16 placebo patients and 19 of the 21 actively treated patients completed diary cards in full. One patient was non-compliant and one set of diary cards was lost in the post.

All patients in the placebo group had their doses increased to the maintenance volume of $1 \mathrm{ml}$. By 28 May, 18 of the actively treated patients achieved the

TABLE III - Symptoms and individual use of drugs (median values) for the Alutard $S Q(n=19)$ and placebo $(n=16)$ treated groups

\begin{tabular}{|c|c|c|c|c|c|c|}
\hline & \multicolumn{3}{|c|}{ Median scores ${ }^{\star}$} & \multirow[b]{2}{*}{$\begin{array}{l}95 \% \text { Confidence } \\
\text { interval† }\end{array}$} & & \multirow[b]{2}{*}{$\mathrm{p}$ Value } \\
\hline & Alutard SQ & Placebo & $\begin{array}{c}\text { Median } \\
\text { difference }\end{array}$ & & & \\
\hline \multicolumn{7}{|c|}{ Symptoms } \\
\hline Overall total & 360 & 928 & 522 & 238 to 825 & & 0.001 \\
\hline Nasal (total) & 49 & 143 & 82 & 38 to 111 & & 0.02 \\
\hline Blocked nose & 12 & 44 & 28 & 3 to 42 & $0 \cdot 03$ & \\
\hline Runny nose & 11 & 43 & 25 & 5 to 43 & 0.03 & \\
\hline Eyes (total) & 37 & 87 & 55 & 10 to 82 & & $0 \cdot 02$ \\
\hline Streaming eyes & & 5 & 4 & 0 to 9 & 0.02 & \\
\hline Itching eyes & 14 & 50 & 26 & 0 to 41 & 0.06 & \\
\hline Chest & 6 & 12 & 6 & -3 to 27 & & $0 \cdot 07$ \\
\hline Mouth and throat & 2 & 31 & 16 & -1 to 32 & & 0.08 \\
\hline \multicolumn{7}{|c|}{ Use of drugs } \\
\hline $\begin{array}{l}\text { Overall total } \\
\text { Sodium cromoglycate: }\end{array}$ & 129 & 627 & 335 & 178 to 574 & & 0.002 \\
\hline Eye drops & 14 & 128 & 82 & 32 to 196 & & 0.01 \\
\hline Nasal spray & 78 & 232 & 136 & 44 to 234 & & 0.01 \\
\hline Acrivastine & 80 & 174 & 107 & 35 to 178 & & 0.004 \\
\hline Prednisolone $\ddagger$ & & 54 & - & - & & 0.001 \\
\hline
\end{tabular}

* For each patient symptom scores during pollen season ( 15 May to $24 \mathrm{July}$ ) were totalled for both active and placebo groups and compared using the Mann-Whitney U test.

† For differences between medians (placebo minus Alutard SQ)

¥Usage analysed as number of patients requiring prednisolone by Fisher's exact test as only eight placebo patients

(and none of Alutard SQ treated patients) required this drug.
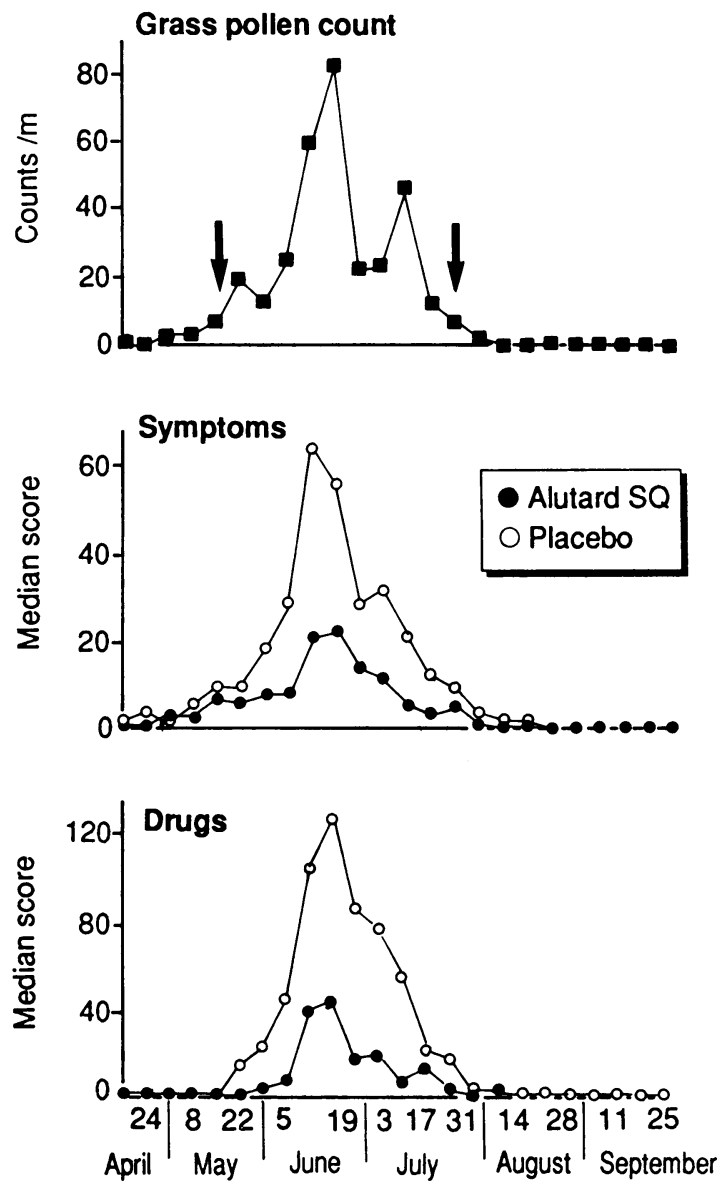

FIG 1-Average weekly grass pollen counts (1989), and symptom and drug scores for Alutard $S Q$ and placebo groups. Arrows represent the beginning (15 May) and end (25 Fuly) of pollen season. Data between these time points were used to calculate confidence intervals and $p$ values for symptoms and drug scores in table III

maintenance dose ( 30000 biological units in $1 \mathrm{ml})$. Of the remaining three patients, one had her maintenance dose limited to $0.6 \mathrm{ml}$ ( 18000 biological units) by large delayed local reactions and two had theirs limited to $0.4 \mathrm{ml}$ (12000 biological units) by the onset of the pollen season. A total of 914 injections (523 active and 391 placebo) were given from April to November 1989, when the study was ended. The greater numbers of injections with active material reflected the difference in numbers between the two groups.

\section{CLINICAL EFFICACY}

Before the pollen season there were no significant differences between the actively treated and placebo groups for symptoms recorded in diaries, drug scores, conjunctival tests, or the size of the immediate and late skin respones to allergen provocation. For both groups the appearance of symptoms and requirement for drugs followed the increase in the average weekly grass pollen count (fig 1). Symptom scores peaked on 12 June, one week before the peak in drug scores, which coincided with the peak pollen count (19 June). Table III shows the results of the effect of Alutard SQ and placebo on symptom and drug scores with $95 \%$ confidence intervals for median differences for all comparisons. For the entire grass pollen season the median scores (median Alutard $v$ median placebo $(95 \%$ confidence intervals for difference between medians)) for both total symptoms $(p=0.001)$ and total medication $(p=0.002)$ were significantly lower in the Alutard SQ treated patients ( $360 v 928(238$ to 825$)$ and $129 v 627$ (178 to 574) respectively) (fig 1). The group treated with Alutard SQ had more symptom free days (29 days) than the placebo group (eight days, $p=0.04$ ( $95 \%$ confidence interval -26 to -1$)$ ).

There was a significant reduction in total nasal 


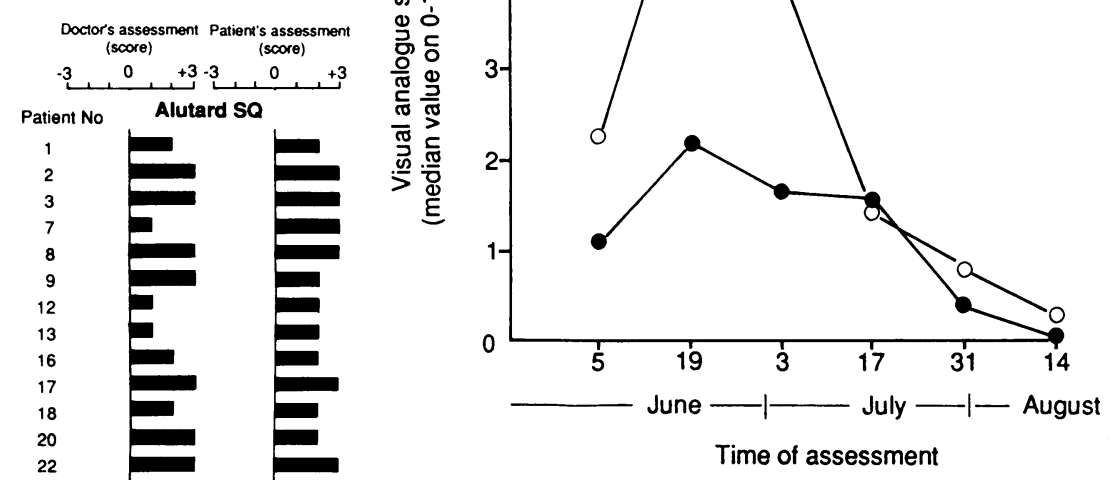

FIG 2-Visual analogue scores for Alutard SQ (closed circle) and placebo (open circle) groups. The points represent the median two weekly visual analogue scores compared by Mann-Whitney $U$ test. The number of patients assessed (Alutard $S Q$, placebo respectively) was 5 fune $(20,15), 19$ fune $(18,12), 3$ fuly $(13,14), 17$ fuly $(14$, 16), 31 fuly $(15,10), 14$ August $(12,9)$

symptoms $(\mathrm{p}=0 \cdot 02,49 v 143$ (38 to 111$))$, blocked nose $(\mathrm{p}=0.03,12 v 44$ ( 3 to 42$))$, runny nose $(\mathrm{p}=0.03,11 v$ 43 (5 to 43$))$, total eye symptoms $(\mathrm{p}=0.02,37 v 87(10$ to 82$)$ ), and streaming eyes ( $\mathrm{p}=0.02,0 v 5$ (0 to 9)) when Alutard SQ was compared with placebo. Itching eyes, chest symptoms, and mouth and throat symptoms were also reduced, but these differences were of only borderline significance. Drug use was significantly reduced in the Alutard SQ group: sodium cromoglycate eye drops $(\mathrm{p}=0.01,14 v 128$ (32 to 196$))$, sodium cromoglycate nasal spray $(\mathrm{p}=0.01,78 v 232$ (44 to $234)$ ), and acrivastine ( $\mathrm{p}=0 \cdot 004,80 v 174$ (35 to 178)). The need for oral prednisolone (nine courses in eight patients) and inhaled salbutamol (two patients) was confined to the placebo group.

At the peak of the season visual analogue scores (fig 2) were lower in the Alutard group. For 19 June ( $p=$ $0.02,2.2$ v $5.5(-4.8$ to -0.5$))$ there was a $60 \%$ decrease in scores (Alutard minus placebo group) and on 3 July a $59 \%$ decrease $(\mathrm{p}=0.01,1 \cdot 7 v 4 \cdot 0(-5 \cdot 0$ to $-1)$ ) (Alutard minus placebo).

The changes in conjunctival allergen sensitivity and the immediate and late skin responses are shown in table IV. Differences in favour of the actively treated patients were found for all three measurements.

Figure 3 shows the patients' and doctor's assessment after the season for each patient. The results showed improvement $(+3$ median value in the active group

TABLE IV-Results of conjunctival and skin provocation tests (median values) before and after immunotherapy

\begin{tabular}{|c|c|c|c|c|}
\hline \multirow[b]{2}{*}{ Provocation test } & \multicolumn{3}{|c|}{ Treatment group } & \multirow[b]{2}{*}{$\mathrm{p}$ Value } \\
\hline & $\begin{array}{c}\text { Alutard SQ } \\
(\mathrm{n}=21)\end{array}$ & $\begin{array}{l}\text { Placebo } \\
(\mathrm{n}=16)\end{array}$ & $\begin{array}{c}95 \% \\
\text { Confidence interval }^{\star}\end{array}$ & \\
\hline \multicolumn{5}{|c|}{ Conjunctival (biological units/ml) } \\
\hline Before immunotherapy & 300 & 300 & & \\
\hline After immunotherapy & 10000 & 1000 & & \\
\hline Median difference & 9900 & & 9700 to 2900 & $<0.001$ \\
\hline \multicolumn{5}{|c|}{$\operatorname{Skin}(\mathrm{mm})$} \\
\hline Immediate $(15 \mathrm{~min}):$ & & & & \\
\hline Before immunotherapy & $19 \cdot 5$ & $21 \cdot 75$ & & \\
\hline After immunotherapy & $11 \cdot 7$ & $19 \cdot 5$ & & \\
\hline Median difference & $-7 \cdot 5$ & $-3 \cdot 5$ & -9 to -0.5 & $0 \cdot 02$ \\
\hline \multicolumn{5}{|l|}{ Late ( 24 hour): } \\
\hline Before immunotherapy & $68 \cdot 5$ & $56 \cdot 7$ & & \\
\hline After immunotherapy & $25 \cdot 1$ & $62 \cdot 9$ & & \\
\hline Median difference & $-36 \cdot 5$ & +14.9 & $-64 \cdot 5$ to -33 & $<0.001$ \\
\hline
\end{tabular}

^ For median differences in effects of immunotherapy for Alutard SQ group minus placebo group. † By Mann-Whitney U test.

Median values given for conjunctival provocation test (provocation concentration of allergen that caused conjunctival itching and redness) and for skin provocation test (size of immediate and late skin responses) before and after pollen season. with +1 in the placebo group); the doctor's median score was +3 and 0 respectively.

\section{SIDE EFFECTS}

Tiredness after injections was commonly reported in both groups. Local reactions of less than $8 \times 8 \mathrm{~cm}$ occurred in both groups, but in 523 injections, 22 reactions greater than $8 \times 8 \mathrm{~cm}$ occurred in the active group, 10 of which were in one patient. The patients and coordinator were not told of their meaning or of any adjustments in dose to ensure blinding of the study. The local reactions were not troublesome, and no treatment was required. Four systemic reactions occurred (two in the active and two in the placebo group) during the induction phase of treatment. One reaction was immediate (within 10 minutes) and three delayed (after two hours). This immediate anaphylactic reaction consisted of flushing and chest tightness and responded rapidly to intramuscular adrenaline. The three dlayed reactions consisted of one case of urticaria at $2 \frac{1}{2}$ hours in an actively treated patient, and two episodes of shortness of breath and dizziness at four hours in a placebo treated patient, which were presumed secondary to hyperventilation.

\section{Discussion}

In this placebo controlled study immunotherapy with an extract of Phleum pratense was effective in reducing symptoms and requirements for drugs in selected patients with severe hay fever. Clinical improvement was accompanied by decreased target organ (conjunctival) and skin sensitivity.

The high sensitivity of the patients as a group was evident by appreciable symptoms even when the average pollen count was low $\left(7\right.$ grains $\left./ \mathrm{m}^{3}\right)$. The clinical trial was therefore conducted with high allergen exposure in very allergic patients. We found clinical improvement after only eight weeks of treatment with the probability, as shown in other studies, ${ }^{11}$ that benefit would continue with further treatment. ${ }^{3}$ The placebo group had high symptom scores, even with maximal medication, which in some patients included oral prednisolone. A short course of oral prednisolone is a useful rescue treatment for severe symptoms and preferable to injected steroids, in which dose adjustments (titration or discontinuation) are not possible.

As emphasised by the Committee on Safety of Medicines (1986) report, ${ }^{5}$ the potential benefit of this treatment must be weighed against its known risks. From our results we conclude that the symptomatic improvement found in the 21 patients justified its use. From 523 injections of active material there were two systemic reactions (one immediate anaphlaxis and one mild delayed urticaria). Both were easily treated and did not lead to the patient dropping out of the study. Although the immediate reaction was recognised quickly and responded promptly to treatment, general anaphylaxis is always a danger so we recommend that immunotherapy should be carried out only in specialised centres. In contrast, delayed local reactions are a well known feature of immunotherapy. They are mild, of no clinical importance, require no treatment, and often go unnoticed by the patient unless specifically asked.

Alutard SQ is widely used in Scandinavia and the rest of Europe. As of 24 September 1990 the Committee on Safety of Medicines had received no reports specifically citing Alutard SQ in association with anaphylaxis. As a depot preparation, Alutard is associated with a lower incidence of systemic reactions, although acute anaphylactic reactions occur as quickly with depot extracts as with aqueous extracts. ${ }^{+}$As a result it has been standard procedure in Europe and world wide to observe patients for 30 minutes unless 
otherwise indicated. ${ }^{34}$ From the results of this study a definite statement on the committee's "two hour rule" is not possible based on the relatively low total numbers of injections. However, the single anaphylactic reaction occurred well within this generally accepted 30 minute observation period. Previous studies suggest that virtually all serious delayed reactions occurred in asthmatic patients, ${ }^{51718}$ so if these patients are specifically excluded a two hour wait for all patients after each injection seems unnecessary.

Thus patient selection for immunotherapy is of the utmost importance. Injections should be given only where there is access to emergency drugs (especially adrenaline) and other resuscitative facilities. People giving the injections should be trained or be under the supervision of doctors trained in immunotherapy. Under these conditions any reactions would be self evident within a very short time after the injection if they were likely to be life threatening.

We conclude that injection immunotherapy with a biologically standardised extract in patients with severe pollen allergy should be considered in those who fail to respond adequately to conventional drug treatment. Patients should be carefully selected and the procedure carried out in a specialised centre.

We thank Allergologisk Laboratorium (ALK Denmark) for supplying Alutard SQ and placebo treatment packs, the Wellcome Foundation for supplying acrivastine $8 \mathrm{mg}$ capsules (Semprex), and Miss Jane Norris-Hill (Pollen Research Project, Polytechnic of North London) for the pollen counts. We particularly thank our patients for their unfailing commitment to the study and their good humour.
1 Fleming DM, Crombie DL. Prevalence of asthma and hay fever in England and Wales. BMF 1987;294:279-83.

2 Howarth PH. Allergic rhinitis: a rational choice of treatment. Respir Med 1989;83:179-88.

3 World Health Organisation/International Union of Immunological Societie Working Group. Current status of allergen immunotherapy. Shortened version of a World Health Organisation/International Union of Immunological Societies Working Group report. Lancet 1989;i:259-61.

4 Malling H-J. Immunotherapy position paper. Allergy 1988;43 (suppl 6):9-31.

5 Anonymous. CSM update. Desensitising vaccines. BMJ 1986;293:948.

6 Frankland AW, Augustin R. Prophylaxis of summer hay-fever and asthma. A controlled trial comparing crude grass-pollen extracts with the isolated main controlled trial comparing crude grass-poll

$7 \mathrm{Kerr} \mathrm{JW}$, Murchison LE. A controlled trial of pollen adsorbate in the treatment of hay fever. Scott Med F 1963;8:485.
.

8 Miller ACML, Tees EC. A metabolizable adjuvant: clinical trial of grass pollen-tyrosine adsorbate. Clin Allergy 1974;4:49-55.

9 Osterballe O. Nasal and skin sensitivity during immunotherapy with two major allergens 19,25 and partially purified extract of timothy grass pollen Allergy 1982;37:169-77.

10 Osterballe $\mathrm{O}$. Immunotherapy in hay fever with two major allergens 19,25 an partially purified extract of timothy grass pollen. A controlled double-blin study. In vivo variables. Season 1. Allergy 1980;35:473-89.

11 Mosbech H, Osterballe O. Does the effect of immunotherapy last after termination of treatment? Follow-up study in patients with grass pollen rhinitis. Allergy 1988;43:523-9.

12 Juniper EF, Frith PA, Dunnett C, Cockcroft DW, Hargreave FE. Reproducibility and correlation of responses to inhaled histamine and methacholine. Thorax 1978;33:705-10.

13 Gjesing B, Jaeger L, Marsh DG, Lowenstein H. The international collaborative study establishing the first international standard for timothy (Phleum study establishing the first international standard for timothy (Phleum 67.

14 Moller C, Bjorksten B, Nilsson G, Dreborg S. The precision of the conjunctival provocation test. Allergy 1984;39:37.

15 Armitage P, Berry G. Distribution free methods. In: Statistical methods in medical research, 2nd ed. Oxford: Blackwell Scientific Publications, 1987: 408-20.

16 Armitage P, Berry G. Statistical inference. In: Statistical methods in medical research. 2nd ed. Oxford: Blackwell Scientific Publications, 1987:93-140.

17 Rawlins MD, Wood SM, Mann RD. Hazards with desensitising vaccines. In: Kurth R, ed. Regulatory control and standardization of allergenic extracts Stuttgart: Springer Verlag, 1988:147-51. (5th international Paul Ehrlich seminar, September 1987.)

18 Lockey RF, Benedict LM, Turkeltaub PC, Bukantz SC. Fatalities from immunotherapy (IT) and skin testing (ST). $f$ Allergy Clin Immunol immunotherapy

(Accepted 21 November 1990)
Margaret Pyke Centre for the Study and Training of Family Planning, London WIV 5TW

G E Robinson, MRCOG, research fellow

W Bounds, SCM, research coordinator

J Guillebaud, FRCOG,

medical director

Haematology Department,

University College and

Middlesex School of

Medicine, London W1

T Burren, BSC, research

assistant

I J Mackie, BSc, lecturer

$\mathrm{K}$ Walshe, FIMLS, senio

medical laboratory scientific

officer

R Faint, BSC, research

assistant

S J Machin, FRCPATH, reader

Correspondence to:

Mr J Guillebaud, Margaret

Pyke Centre, 15 Batemans

Buildings, Soho Square,

London W1V 5TW.

BMF 1991;302:269-71

\title{
Changes in haemostasis after stopping the combined contraceptive pill: implications for major surgery
}

\author{
G E Robinson, T Burren, I J Mackie, W Bounds, K Walshe, R Faint, J Guillebaud, S J Machin
}

\section{Abstract}

Objective-To investigate the changes in haemostasis in the three months immediately after stopping the combined contraceptive pill.

Design-Prospective randomised study.

Setting-Family planning centre in London.

Subjects -24 women aged $35-45$ investigated before, during, and after six months' use of combined oral contraceptives containing $30 \mu \mathrm{g}$ ethinyl oestradiol together with the progestogens desogestrel or gestodene.

Main outcome measures and results-Blood samples were taken immediately before and after six months of oral contraceptive use and one, two, four, six, eight, and 12 weeks after the pill had been stopped. During the six months of oral contraceptive use the plasma concentration of factor $X$ and fibrinogen increased and that of antithrombin III decreased. Between two and six weeks after stopping the pill a rebound phenomenon occurred with plasma concentrations of antithrombin III increasing (mean change from baseline at two weeks $0.06 \mathrm{IU} / 1$ and at six weeks $0 \cdot 10 \mathrm{IU} / \mathrm{l}$ ) and fibrinogen decreasing $(0.26 \mathrm{~g} / \mathrm{l}$ change at two weeks and $0.40 \mathrm{~g} / 1$ at six weeks). Factor $\mathbf{X}$ concentrations fell gradually and the values at eight weeks were not significantly different from those found before the combined pill was started.

Conclusion-The combined pill should be stopped at least four weeks before major surgery, which carries the risk of postoperative thrombosis, to allow the potentially prothrombotic haemostatic changes that occur during its use to be corrected.

\section{Introduction}

The introduction and widespread use of the combined oral contraceptive has been associated with an increase in venous thromboembolism. Epidemiological studies conducted mainly in the 1960s and early 1970 s suggest that this increased risk (approximately fourfold) is unrelated to duration of oral contraceptive use or smoking habits. ${ }^{1}$ The reduction in the dose of ethinyloestradiol in combined pill preparations has, however, been shown to be associated with a reduction in morbidity from venous thromboembolism, ${ }^{2}$ implicating the oestrogen component of the oral contraceptive in the pathogenesis of thromboembolism.

Several studies have shown that women taking combined oral contraceptives show changes in haemostasis. The plasma concentrations of the coagulation factors I (fibrinogen), II, VII, IX, X, and XII have consistently been reported to increase ${ }^{3-5}$ together with an associated decrease in antithrombin III concentration. ${ }^{45}$ These changes may predispose to venous thromboembolism, especially if not balanced by an increase either in fibrinolytic activity or of other inhibitory proteins of the coagulation cascade, such as protein C.

Major surgery is a recognised risk factor for venous 